\title{
Liu Estimator in Semiparametric Partially Linear Varying Coefficient Models
}

\author{
Jing $\mathrm{Li}^{1}$, \& Xueyan $\mathrm{Li}^{2}$ \\ ${ }^{1}$ School of Applied Technology, China University of Labor Relations, Beijing 100048, P.R.China \\ ${ }^{2}$ School of Science, Civil Aviation University of China, Tianjin, 300300, P.R.China \\ Correspondence: Jing Li, School of Applied Technology, China University of Labor Relations, Beijing 100048, P.R.China. \\ E-mail: lijjingg@163.com
}

Received: October 11, 2019 Accepted: November 6, 2019 Online Published: November 13, 2019

doi:10.5539/ijsp.v8n6p69 URL: https://doi.org/10.5539/ijsp.v8n6p69

\begin{abstract}
This paper considers biased estimation for partially linear varying coefficient model to overcome the problem of multicollinearity. By the Liu estimation approach, we construct a profile Liu estimator for the constant coefficients. Furthermore, a restricted profile-Liu estimator is proposed for the situation that some additional linear restrictions are available. The properties of the proposed estimators are investigated.
\end{abstract}

Keywords: Liu estimation, partially linear varying coefficient model, profile least-squares, restricted estimation

\section{Introduction}

Over the last three decades, nonparametric and semiparametric models have attracted much attention. We consider the following semiparametric partially linear varying coefficient model

$$
y_{i}=\mathbf{x}_{i}^{\mathrm{T}} \boldsymbol{\beta}+\mathbf{z}_{i}^{\mathrm{T}} \boldsymbol{\alpha}\left(u_{i}\right)+\varepsilon_{i}, i=1,2, \cdots, n
$$

with $y_{i}^{\prime} s$ are responses; $\mathbf{x}_{i}=\left(x_{i 1}, x_{i 2}, \cdots, x_{i p}\right)^{\mathrm{T}}, \mathbf{z}_{i}=\left(z_{i 1}, z_{i 2}, \cdots, z_{i q}\right)^{\mathrm{T}}$ and $u_{i}$ are associated covariates; $\boldsymbol{\beta}=\left(\beta_{1}, \beta_{2}, \cdots, \beta_{p}\right)^{\mathrm{T}}$ is a vector of unknown regression coefficients and $\alpha(\cdot)=\left(\alpha_{1}(\cdot), \alpha_{1}(\cdot), \cdots, \alpha_{q}(\cdot)\right)^{\mathrm{T}}$ are unknown nonparametric functions; $\varepsilon_{i}^{\prime} s$ are independent random errors with $\mathrm{E}\left(\varepsilon_{i} \mid \mathbf{x}_{i}, \mathbf{z}_{i}, u_{i}\right)=0$ and $\sigma^{2}=\mathrm{E}\left[\varepsilon_{i}^{2} \mid \mathbf{x}_{i}, \mathbf{z}_{i}, u_{i}\right]$.

For model (1.1), how to estimate the unknown regression coefficients is an interesting and important problem. Several methods have been proposed, such as local polynomial estimator of Zhang et al. (2002), series estimator of Ahmad et al. (2005), and local linear based profile least-squares approach of Fan and Huang (2005). However, the problem of multicollinearity was not considered by the above literatures. The problem of multi-collinearity of linear regression model may cause serious problem for least squares estimators of the regression coefficients. To solve this problem, some biased estimators including ridge estimator and Liu estimator as well as principal components regression estimator have been proposed.

In the last two decades, biased estimation in semiparametric models has received much attention. For partially linear models, Hu (2005), Akdeniz and Tabakan (2009) and Roozbeh and Arashi (2013) proposed the ridge estimators of the parametric components. Akdeniz and Akdeniz Duran (2010) developed a Liu-type estimator. For partially linear additive models, Wei and Wang (2016) proposed Liu estimator and restricted Liu estimator for the parametric components. For partially linear varying coefficient model (1.1), Liu et al. (2017) constructed a stochastic restricted ridge estimator for the parametric components. Following the idea of Akdeniz and Akdeniz Duran (2010) and Wei and Wang (2016), we will construct Liu estimator for the parametric component $\beta$.

The Liu estimator is constructed in Section 2. In Section 3, the restricted Liu estimator is proposed. Conclusion is given in Section 4. Section 5 offers the proofs of the main results.

\section{Liu Estimator of the Parametric Components}

To apply the Liu estimation approch for model (1.1), we first introduce profile least-squares approach.

If $\boldsymbol{\beta}$ is known, then model (1.1) can be rewritten as the following varying coefficient model

$$
y_{i}^{*}=\alpha_{1}\left(u_{i}\right) z_{i 1}+\cdots+\alpha_{q}\left(u_{i}\right) z_{i q}+\varepsilon_{i}, \quad i=1,2, \cdots, n
$$

with $y_{i}^{*}=y_{i}-\mathbf{x}_{i}^{\mathrm{T}} \boldsymbol{\beta}$. The local linear method was applied to estimate the varying coefficient functions of model (2.1). For 
$u$ in a small neighborhood of $u_{0}$, then we have

$$
\alpha_{j}(u) \approx \alpha_{j}\left(u_{0}\right)+\alpha_{j}^{\prime}\left(u_{0}\right)\left(u-u_{0}\right) \quad j=1,2, \cdots, q
$$

Then, the estimators of $\left\{\left(\alpha_{j}\left(u_{0}\right), \alpha_{j}^{\prime}\left(u_{0}\right)\right), \quad j=1,2, \cdots, q\right\}$ can be obtained by minimizing

$$
\sum_{i=1}^{n}\left[y_{i}^{*}-\sum_{j=1}^{q}\left\{\alpha_{j}\left(u_{0}\right)+\alpha_{j}^{\prime}\left(u_{0}\right)\left(u-u_{0}\right)\right\} z_{i j}\right]^{2} \mathrm{~K}_{h}\left(u_{i}-u_{0}\right)
$$

where $K$ is a kernel function, $h$ is a bandwidth and $K_{h}(\cdot)=K(\cdot / h) / h$.

Denote

$$
\begin{gathered}
\mathbf{X}=\left[\begin{array}{c}
\mathbf{x}_{1}^{\mathrm{T}} \\
\mathbf{x}_{2}^{\mathrm{T}} \\
\vdots \\
\mathbf{x}_{n}^{\mathrm{T}}
\end{array}\right]=\left[\begin{array}{ccc}
x_{11} & \cdots & x_{1 p} \\
x_{21} & \cdots & x_{2 p} \\
\vdots & \ddots & \vdots \\
x_{n 1} & \cdots & x_{n p}
\end{array}\right], \mathbf{Z}=\left[\begin{array}{c}
\mathbf{z}_{1}^{\mathrm{T}} \\
\mathbf{z}_{2}^{\mathrm{T}} \\
\vdots \\
\mathbf{z}_{n}^{\mathrm{T}}
\end{array}\right]=\left[\begin{array}{ccc}
z_{11} & \cdots & z_{1 q} \\
z_{21} & \cdots & z_{2 q} \\
\vdots & \ddots & \vdots \\
z_{n 1} & \cdots & z_{n q}
\end{array}\right], \\
\mathbf{Y}=\left[\begin{array}{c}
y_{1} \\
y_{2} \\
\vdots \\
y_{n}
\end{array}\right], \mathbf{M}=\left[\begin{array}{c}
\mathbf{z}_{1}^{\mathrm{T}} \boldsymbol{\alpha}\left(u_{1}\right) \\
\mathbf{z}_{2}^{\mathrm{T}} \boldsymbol{\alpha}\left(u_{2}\right) \\
\vdots \\
\mathbf{z}_{n}^{\mathrm{T}} \boldsymbol{\alpha}\left(u_{n}\right)
\end{array}\right], \mathbf{D}_{u_{0}}=\left[\begin{array}{cc}
\mathbf{z}_{1}^{\mathrm{T}} & \mathbf{z}_{1}^{\mathrm{T}} \frac{u_{1}-u_{0}}{h} \\
\mathbf{z}_{2}^{\mathrm{T}} & \mathbf{z}_{2}^{\mathrm{T}} \frac{u_{2}-u_{0}}{h} \\
\vdots & \vdots \\
\mathbf{z}_{n}^{\mathrm{T}} & \mathbf{z}_{n}^{\mathrm{T}} \frac{u_{n}-u_{0}}{h}
\end{array}\right]
\end{gathered}
$$

and

$$
\boldsymbol{\varepsilon}=\left(\varepsilon_{1}, \cdots, \varepsilon_{n}\right)^{\mathrm{T}}, \mathbf{W}_{u_{0}}=\operatorname{diag}\left\{K_{h}\left(u_{1}-u_{0}\right), K_{h}\left(u_{2}-u_{0}\right), \cdots, K_{h}\left(u_{n}-u_{0}\right)\right\} .
$$

Then varying coefficient model (2.1) can be written as

$$
\mathbf{Y}-\mathbf{X} \boldsymbol{\beta}=\mathbf{M}+\varepsilon
$$

The solution the problem (2.3) is given by

$$
\left[\hat{\alpha}_{1}\left(u_{0}\right), \cdots, \hat{\alpha}_{p}\left(u_{0}\right), h \hat{\alpha}_{1}^{\prime}\left(u_{0}\right), \cdots, h \hat{\alpha}_{p}^{\prime}\left(u_{0}\right)\right]^{\mathrm{T}}=\left\{\mathbf{D}_{u_{0}}^{\mathrm{T}} \mathbf{W}_{u_{0}} \mathbf{D}_{u_{0}}\right\}^{-1} \mathbf{D}_{u_{0}}^{\mathrm{T}} \mathbf{W}_{u_{0}}(\mathbf{Y}-\mathbf{X} \boldsymbol{\beta}),
$$

We can define the estimator of $\mathbf{M}$ as

$$
\overline{\mathbf{M}}=\mathbf{S}(\mathbf{Y}-\mathbf{X} \boldsymbol{\beta})
$$

where

$$
\mathbf{S}=\left[\begin{array}{c}
\left(\mathbf{z}_{1}^{\mathrm{T}} \mathbf{0}\right)\left\{\mathbf{D}_{u_{1}}^{\mathrm{T}} \mathbf{W}_{u_{1}} \mathbf{D}_{u_{1}}\right\}^{-1} \mathbf{D}_{u_{1}}^{\mathrm{T}} \mathbf{W}_{u_{1}} \\
\left(\mathbf{z}_{2}^{\mathrm{T}} \mathbf{0}\right)\left\{\mathbf{D}_{u_{2}}^{\mathrm{T}} \mathbf{W}_{u_{2}} \mathbf{D}_{u_{2}}\right\}^{-1} \mathbf{D}_{u_{2}}^{\mathrm{T}} \mathbf{W}_{u_{2}} \\
\vdots \\
\left(\mathbf{z}_{n}^{\mathrm{T}} \mathbf{0}\right)\left\{\mathbf{D}_{u_{n}}^{\mathrm{T}} \mathbf{W}_{u_{n}} \mathbf{D}_{u_{n}}\right\}^{-1} \mathbf{D}_{u_{n}}^{\mathrm{T}} \mathbf{W}_{u_{n}}
\end{array}\right] .
$$

Replacing $\mathbf{M}$ of (2.4) by $\overline{\mathbf{M}}$, we can get the following linear regression model

$$
\overline{\mathbf{Y}}=\overline{\mathbf{X}} \boldsymbol{\beta}+\boldsymbol{\varepsilon} .
$$

where $\overline{\mathbf{Y}}=\left(\mathbf{I}_{n}-\mathbf{S}\right) \mathbf{Y}, \overline{\mathbf{X}}=\left(\mathbf{I}_{n}-\mathbf{S}\right) \mathbf{X}$. Then, the profile least-squares (PLS) estimator for parametric component $\boldsymbol{\beta}$ can be obtained as

$$
\hat{\boldsymbol{\beta}}_{\mathrm{P}}=\arg \min _{\boldsymbol{\beta} \in R^{p}}\left[(\overline{\mathbf{Y}}-\overline{\mathbf{X}} \boldsymbol{\beta})^{\mathrm{T}}(\overline{\mathbf{Y}}-\overline{\mathbf{X}} \boldsymbol{\beta})\right]=\left(\overline{\mathbf{X}}^{\mathrm{T}} \overline{\mathbf{X}}\right)^{-1} \overline{\mathbf{X}}^{\mathrm{T}} \overline{\mathbf{Y}}
$$

Based on the linear model (2.6) and the Liu estimation approach, we define the following objective function:

$$
F_{1}(\boldsymbol{\beta})=(\overline{\mathbf{Y}}-\overline{\mathbf{X}} \boldsymbol{\beta})^{\mathrm{T}}(\overline{\mathbf{Y}}-\overline{\mathbf{X}} \boldsymbol{\beta})+\left(d \hat{\boldsymbol{\beta}}_{\mathrm{P}}-\boldsymbol{\beta}\right)^{\mathrm{T}}\left(d \hat{\boldsymbol{\beta}}_{\mathrm{P}}-\boldsymbol{\beta}\right) .
$$

Then, we have

$$
\frac{\partial F_{1}(\boldsymbol{\beta})}{\partial \boldsymbol{\beta}}=-2 \overline{\mathbf{X}}^{\mathrm{T}} \overline{\mathbf{Y}}+2 \overline{\mathbf{X}}^{\mathrm{T}} \overline{\mathbf{X}} \boldsymbol{\beta}-2 d \hat{\boldsymbol{\beta}}_{\mathrm{P}}+2 \boldsymbol{\beta}=\mathbf{0} .
$$

We can obtain the following profile least-squares Liu estimator by solving equation (2.9) as

$$
\hat{\boldsymbol{\beta}}_{\mathrm{PL}}(d)=\left(\overline{\mathbf{X}}^{\mathrm{T}} \overline{\mathbf{X}}+\mathbf{I}_{\mathrm{p}}\right)^{-1}\left(\overline{\mathbf{X}}^{\mathrm{T}} \overline{\mathbf{Y}}+d \hat{\boldsymbol{\beta}}_{\mathrm{P}}\right) .
$$


For $\hat{\boldsymbol{\beta}}_{\mathrm{PL}}(d)$, we have the following properties.

Theorem 2.1 For the profile least-squares Liu estimator $\hat{\boldsymbol{\beta}}_{\mathrm{PL}}\left(\right.$ d), denote $\mathbf{M}=\mathbf{m}_{1}+\mathbf{m}_{2}$, we have

$$
\operatorname{Bias}\left(\hat{\boldsymbol{\beta}}_{\mathrm{PL}}(d)\right)=-(1-d)\left(\overline{\mathbf{X}}^{\mathrm{T}} \overline{\mathbf{X}}+\mathbf{I}_{\mathrm{p}}\right)^{-1} \boldsymbol{\beta}+\boldsymbol{\Delta} \overline{\mathbf{X}}^{\mathrm{T}}\left(\mathbf{I}_{n}-\mathbf{S}\right) \mathbf{M}, \quad \operatorname{Cov}\left(\hat{\boldsymbol{\beta}}_{\mathrm{PL}}(d)\right)=\boldsymbol{\Delta} \boldsymbol{\Phi} \boldsymbol{\Delta}^{\mathrm{T}},
$$

where $\boldsymbol{\Delta}=\left(\overline{\mathbf{X}}^{\mathrm{T}} \overline{\mathbf{X}}+\mathbf{I}_{\mathrm{p}}\right)^{-1}\left(\overline{\mathbf{X}}^{\mathrm{T}} \overline{\mathbf{X}}+d \mathbf{I}_{\mathrm{p}}\right)\left(\overline{\mathbf{X}}^{\mathrm{T}} \overline{\mathbf{X}}\right)^{-1}, \mathbf{\Phi}=\sigma^{2} \overline{\mathbf{X}}^{\mathrm{T}}\left(\mathbf{I}_{n}-\mathbf{S}\right)\left(\mathbf{I}_{n}-\mathbf{S}\right)^{\mathrm{T}} \overline{\mathbf{X}}$.

Theorem 2.2 Under the assumptions 1-5 in Section 5, $\boldsymbol{\beta}_{\mathrm{PL}}(d)$ is asymptotically normal, namely,

$$
\sqrt{n}\left(\hat{\boldsymbol{\beta}}_{\mathrm{PL}}(d)-\boldsymbol{\beta}\right) \stackrel{D}{\longrightarrow} N\left(\mathbf{0}, \sigma^{2} \boldsymbol{\Sigma}^{-1}\right),
$$

where $\boldsymbol{\Sigma}=\mathrm{E}\left(\mathbf{X X}^{\mathrm{T}}\right)-\mathrm{E}\left[\boldsymbol{\Phi}(U) \boldsymbol{\Gamma}^{-1}(U) \boldsymbol{\Phi}(U)^{\mathrm{T}}\right], \mathbf{\Gamma}(U)=\mathrm{E}\left[\mathbf{Z Z}^{\mathrm{T}} \mid U\right], \boldsymbol{\Phi}(U)=\mathrm{E}\left[\mathbf{X}^{\mathrm{T}} \mathbf{Z} \mid U\right]$.

\section{Restricted Profile Least-Squares Liu Estimator}

In this section, we consider the following additional restrictions on the parametric component

$$
\mathbf{A} \beta=\mathbf{b},
$$

with $\mathbf{A}$ is a $k \times p$ matrix of known constants, $\mathbf{b}$ is a $k$-vector of known constants, we assume that $\operatorname{rank}(\mathbf{A})=k$.

Based on the linear model (2.6) with restriction conditions (3.1), we define the following Lagrange function

$$
F_{2}(\boldsymbol{\beta}, \lambda)=(\overline{\mathbf{Y}}-\overline{\mathbf{X}} \boldsymbol{\beta})^{\mathrm{T}}(\overline{\mathbf{Y}}-\overline{\mathbf{X}} \boldsymbol{\beta})+\left(d \hat{\boldsymbol{\beta}}_{\mathrm{P}}-\boldsymbol{\beta}\right)^{\mathrm{T}}\left(d \hat{\boldsymbol{\beta}}_{\mathrm{P}}-\boldsymbol{\beta}\right)+2 \lambda^{\mathrm{T}}(\mathbf{A} \boldsymbol{\beta}-\mathbf{b}) .
$$

By differentiating $F_{2}$ with respect to $\beta$ and $\lambda$, then we obtain the following system of vector equations:

$$
\left\{\begin{array}{l}
\frac{\partial F_{3}(\boldsymbol{\beta}, \lambda)}{\partial \boldsymbol{\beta}}=-2 \overline{\mathbf{X}}^{\mathrm{T}} \overline{\mathbf{Y}}+2 \overline{\mathbf{X}}^{\mathrm{T}} \overline{\mathbf{X}} \boldsymbol{\beta}-2 d \hat{\boldsymbol{\beta}}_{\mathrm{P}}+2 \boldsymbol{\beta}+2 \mathbf{A}^{\mathrm{T}} \boldsymbol{\lambda}=\mathbf{0}, \\
\frac{\partial F_{3}(\boldsymbol{\beta}, \lambda)}{\partial \lambda}=2(\mathbf{A} \boldsymbol{\beta}-\mathbf{b})=\mathbf{0} .
\end{array}\right.
$$

Solving the above equations, the restricted profile least-squares Liu estimator of $\beta$ is obtained as

$$
\hat{\boldsymbol{\beta}}_{\mathrm{PL}}^{\mathrm{R}}=\hat{\boldsymbol{\beta}}_{\mathrm{PL}}(d)-\left(\overline{\mathbf{X}}^{\mathrm{T}} \overline{\mathbf{X}}+\mathbf{I}_{\mathrm{p}}\right)^{-1} \mathbf{A}^{\mathrm{T}}\left[\mathbf{A}\left(\overline{\mathbf{X}}^{\mathrm{T}} \overline{\mathbf{X}}+\mathbf{I}_{\mathrm{p}}\right)^{-1} \mathbf{A}^{\mathrm{T}}\right]^{-1}\left[\mathbf{A} \hat{\boldsymbol{\beta}}_{\mathrm{PL}}(d)-\mathbf{b}\right] .
$$

The following theorem gives the asymptotic normality of $\hat{\boldsymbol{\beta}}_{\mathrm{PL}}^{\mathrm{R}}$.

Theorem 3.1 Under the assumptions 1-5 in Section 5, the restricted profile least-squares Liu estimator $\hat{\boldsymbol{\beta}}_{\mathrm{PL}}^{\mathrm{R}}$ is asymptotically normal, namely,

$$
\sqrt{n}\left(\hat{\boldsymbol{\beta}}_{\mathrm{PL}}^{\mathrm{R}}-\boldsymbol{\beta}\right) \stackrel{D}{\longrightarrow} N\left(\mathbf{0}, \sigma^{2} \mathbf{\Omega}\right),
$$

with $\mathbf{\Omega}=\boldsymbol{\Sigma}^{-1}-\boldsymbol{\Sigma}^{-1} \mathbf{A}^{\mathrm{T}}\left(\mathbf{A} \boldsymbol{\Sigma}^{-1} \mathbf{A}^{\mathrm{T}}\right)^{-1} \mathbf{A} \boldsymbol{\Sigma}^{-1}$.

\section{Conclusions}

For partially linear varying coefficient models, this paper proposed a Liu-type biased estimator for the parametric component as well as a restricted Liu estimator for multi-collinearity and linear restrictions. The properties of the proposed estimators are given.

\section{Proof of the Main Results}

We consider the following assumptions of Fan and Huang (2005).

Assumption 1. The random variable $U$ has a bounded support $\Psi$. Its density function $f(\cdot)$ is Lipschitz continuous and bounded away from 0 on its support.

Assumption 2. The $q \times q$ matrix $E\left(\mathbf{Z Z}^{\mathrm{T}} \mid U\right)$ is non-singular for each $U \in \mathbf{\Psi} . E\left(\mathbf{Z Z}^{\mathrm{T}} \mid U\right), E\left(\mathbf{Z Z} \mathbf{Z}^{\mathrm{T}} \mid U\right)^{-1}$ and $E\left(\mathbf{Z X} \mathbf{X}^{\mathrm{T}} \mid U\right)$ are all Lipschitz continuous.

Assumption 3. There is an $s>2$ such that $E\|\mathbf{X}\|^{2 s}<\infty$ and $E\|\mathbf{Z}\|^{2 s}<\infty$ and $n^{2 k-1} h \rightarrow \infty$ for some $k<2-s^{-1}$.

Assumption 4. $\left\{\alpha_{j}(\cdot), j=1, \cdots, q\right\}$ have continuous second derivatives in $U \in \boldsymbol{\Psi}$.

Assumption 5. The function $K(\cdot)$ is a symmetric density function with compact support and the bandwidth $h$ satisfies $n h^{8} \rightarrow 0$ and $n h^{2} /(\log n)^{2} \rightarrow \infty$.

Lemma 5.1 Under the assumptions 1-4, the profile least-squares estimator of $\boldsymbol{\beta}_{\mathrm{P}}$ is asymptotically normal, namely,

$$
\left.\sqrt{n}\left(\hat{\boldsymbol{\beta}}_{\mathrm{P}}-\boldsymbol{\beta}\right) \stackrel{D}{\longrightarrow} N\left(\mathbf{0}, \sigma^{2} \boldsymbol{\Sigma}^{-1}\right)\right)
$$


This lemma is Theorem 4.1 of Fan and Huang (2012).

Proof of Theorem 2.1. By the definition of $\hat{\boldsymbol{\beta}}_{\mathrm{PL}}(d)$, we can get

$$
\begin{aligned}
\hat{\boldsymbol{\beta}}_{\mathrm{PL}}(d) & =\left(\overline{\mathbf{X}}^{\mathrm{T}} \overline{\mathbf{X}}+\mathbf{I}_{\mathrm{p}}\right)^{-1}\left(\overline{\mathbf{X}}^{\mathrm{T}} \overline{\mathbf{Y}}+d \hat{\boldsymbol{\beta}}_{\mathrm{P}}\right)=\left(\overline{\mathbf{X}}^{\mathrm{T}} \overline{\mathbf{X}}+\mathbf{I}_{\mathrm{p}}\right)^{-1}\left(\overline{\mathbf{X}}^{\mathrm{T}} \overline{\mathbf{X}}+d \mathbf{I}_{\mathrm{p}}\right) \hat{\boldsymbol{\beta}}_{\mathrm{P}} \\
& =\boldsymbol{\beta}-(1-d)\left(\overline{\mathbf{X}}^{\mathrm{T}} \overline{\mathbf{X}}+\mathbf{I}_{\mathrm{p}}\right)^{-1} \boldsymbol{\beta}+\boldsymbol{\Delta} \overline{\mathbf{X}}^{\mathrm{T}}\left(\mathbf{I}_{n}-\mathbf{S}\right) \mathbf{M}+\boldsymbol{\Delta} \overline{\mathbf{X}}^{\mathrm{T}}\left(\mathbf{I}_{n}-\mathbf{S}\right) \boldsymbol{\varepsilon} .
\end{aligned}
$$

Then, it is easy to prove that

$$
\operatorname{Bias}\left(\hat{\boldsymbol{\beta}}_{\mathrm{PL}}(d)\right)=-(1-d)\left(\overline{\mathbf{X}}^{\mathrm{T}} \overline{\mathbf{X}}+\mathbf{I}_{\mathrm{p}}\right)^{-1} \boldsymbol{\beta}+\boldsymbol{\Delta} \overline{\mathbf{X}}^{\mathrm{T}}\left(\mathbf{I}_{n}-\mathbf{S}\right) \mathbf{M}, \quad \operatorname{Cov}\left(\hat{\boldsymbol{\beta}}_{\mathrm{PM}}(d)\right)=\Delta \boldsymbol{\Phi} \boldsymbol{\Delta}^{\mathrm{T}} .
$$

Proof of Theorem 2.2. Like the proof of Theorem 2.2 of Wei and Wang (2016), by the definition of $\hat{\boldsymbol{\beta}}_{\mathrm{PL}}(d)$, we can get

$$
\sqrt{n}\left(\hat{\boldsymbol{\beta}}_{\mathrm{PL}}(d)-\boldsymbol{\beta}\right)=\left(\overline{\mathbf{X}}^{\mathrm{T}} \overline{\mathbf{X}}+\mathbf{I}_{\mathrm{p}}\right)^{-1}\left(\overline{\mathbf{X}}^{\mathrm{T}} \overline{\mathbf{X}}+d \mathbf{I}_{\mathrm{p}}\right) \sqrt{n}\left(\hat{\boldsymbol{\beta}}_{\mathrm{P}}-\boldsymbol{\beta}\right)-(1-d) \sqrt{n}\left(\overline{\mathbf{X}}^{\mathrm{T}} \overline{\mathbf{X}}+\mathbf{I}\right)^{-1} \boldsymbol{\beta}
$$

and

$$
\frac{1}{n}\left(\overline{\mathbf{X}}^{\mathrm{T}} \overline{\mathbf{X}}+\mathbf{I}_{\mathrm{p}}\right) \stackrel{p}{\longrightarrow} \boldsymbol{\Sigma}, \quad \frac{1}{n}\left(\overline{\mathbf{X}}^{\mathrm{T}} \overline{\mathbf{X}}+d \mathbf{I}_{\mathrm{p}}\right) \stackrel{p}{\longrightarrow} \boldsymbol{\Sigma}, \quad(1-d) \sqrt{n}\left(\overline{\mathbf{X}}^{\mathrm{T}} \overline{\mathbf{X}}+\mathbf{I}_{\mathrm{p}}\right)^{-1} \boldsymbol{\beta}=O_{p}\left(n^{-1 / 2}\right),
$$

By the Lemma 5.1 and the Slutsky theorem, we have

$$
\sqrt{n}\left(\hat{\boldsymbol{\beta}}_{\mathrm{PL}}(d)-\boldsymbol{\beta}\right) \stackrel{D}{\longrightarrow} N\left(\mathbf{0}, \sigma^{2} \boldsymbol{\Sigma}^{-1}\right) .
$$

Proof of Theorem 3.1. By the similar way in the proof of Theorem 3.1 of Wei and Wang (2016), we can proof Theorem 3.1, we omit the details.

\section{References}

Ahmad, I., Leelahanon, S., \& Li, Q. (2005). Efficient estimation of semiparametric partially linear varying coefficient model. The Annals of Statistics, 33, 258-283. https://dx.doi.org/10.1214/009053604000000931

Akdeniz, F., \& Akdeniz, D. E. (2010). Liu-type estimator in semiparametric regression Model. Journal of Statistical Computation and Simulation, 80, 853-871. https://dx.doi.org/10.1080/00949650902821699

Akdeniz, F., \& Tabakan, G. (2009). Restricted ridge estimators of the parameters in semiparametric regression model. Communications in Statistics-Theory and Methods, 38(11), 1852-1869. https://dx.doi.org/10.1080/03610920802470109

Fan, J. Q., \& Huang, T. (2005). Profile likelihood inferences on semiparametric varying-coefficient partially linear models. Bernoulli, 11(6), 1031-1057. https://dx.doi.org/10.3150/bj/1137421639

Hastie, T., \& Tibshirani, R. (1993). Varying-coefficient models (with discussion). Journal of the Royal Statistical Society: Series B, 55(4), 757-796. https://doi.org/10.1111/j.2517-6161.1993.tb01939.x

Hu, H. (2005). Ridge estimation of a semiparametric regression model. Journal of Computational and Applied Mathematics, 176(1), 215-222. https://doi:10.1016/j.cam.2004.07.032

Liu, C., Wei, J., \& Wei, C. H. (2017). Stochastic Restricted Ridge Regression Estimator in Partially Linear Varying Coefficient Models. MATHEMATICA APPLICATA, 30(4), 774-779.

Rao, C. R., Toutenburg, H., Shalabh, \& Heumann, C. (2008). Linear Models: Least Squares and Alternatives, Springer, Berlin.

Roozbeh, M., \& Arashi, M. (2013). Feasible ridge estimator in partially linear models. Journal of Multivariate Analysis, 116, 35-44. https://doi:10.1016/j.jmva.2012.11.006

Wei, C. H., \& Wang, X. N. (2016). Liu-type estimator in semiparametric partially linear additive models. Journal of Nonparametric Statistics, 28(3), 459-468. https://dx.doi.org/10.1080/10485252.2016.1163357

Zhang,W. Y., Lee, S., \& Song, X. Y. (2002). Local polynomial fitting in semivarying-coefficient model. Journal of Multivariate Analysis, 82, 166-188. https://doi:10.1006/jmva.2001.2012

\section{Copyrights}

Copyright for this article is retained by the author(s), with first publication rights granted to the journal.

This is an open-access article distributed under the terms and conditions of the Creative Commons Attribution license (http://creativecommons.org/licenses/by/4.0/). 